\title{
Set up of a new in vitro model to study dietary fructans fermentation in formula-fed babies
}

\author{
Gwenaëlle Le Blay†, Christophe Chassard, Selina Baltzer and Christophe Lacroix* \\ Laboratory of Food Biotechnology, Institute of Food Science and Nutrition, ETH Zurich, Schmelzbergstrasse 7, 8092 Zurich, \\ Switzerland
}

(Received 18 March 2008 - Revised 15 June 2009 - Accepted 22 June 2009 - First published online 15 September 2009)

A new in vitro fermentation model with immobilised infant faecal microbiota simulating the proximal colon of a formula-fed baby was developed and used to test the effects of known prebiotic fructans. Intestinal fermentation, based on a previously developed colonic fermentation model, using a new feeding medium simulating a formula-fed infant ileal chyme, was carried out for seventy-one consecutive days divided into four stabilisation periods intercalated with four prebiotic treatment periods. At the end of the first stabilisation period, total bacterial concentration in colonised beads and in faecal sample was similar, metabolite concentrations returned to stabilisation values after each treatment period. As expected, the four prebiotic treatments significantly increased the bifidobacterial populations, whereas they decreased bacteroides and clostridia. No difference was observed in the prebiotic effect of these substrates selected. The treatments significantly increased total production of SCFA and decreased ammonia compared to stabilisation periods. Long-term stability of the system together with the reproducibility of the known prebiotic effects highlights the potential of the present model to quantify and compare the effects of different substrates in a formula-fed infant microbiota within the same fermentation experiment.

In vitro colon model: Immobilised faecal microbiota: Formula-fed infants: Prebiotics: Fructans

The intestinal microbiota performs important metabolic and immunological functions and acts as a biological barrier against pathogens. Its composition has been found to vary with age. Initially sterile, the gastrointestinal tract of newborn is rapidly colonised by bacteria from both maternal vaginal and faecal microbiotas and the surrounding environment through a complex process ${ }^{(1)}$. The microbial ecology of the infant gut is influenced by diet among other environmental and genetic factors. It is generally believed that breastfed infants have a microbiota dominated by bifidobacteria and lactobacilli, creating a protective environment against pathogens ${ }^{(2)}$. Formula-fed infants with a more complex microbiota are more susceptible to intestinal disorders $^{(3)}$. Human milk contains high levels of oligosaccharides that stimulate the growth of indigenous bifidobacteria and lactobacilli and may contribute to the protection of breast-fed infants against infections and inflammations ${ }^{(4,5)}$. There is great interest to develop new infant formula sustaining gut microbiota akin to breast-fed infants.

Dietary modulation of the gut microbiota by probiotics and prebiotics is an important feature in nutritional sciences. A prebiotic is a selective fermented ingredient that promotes specific changes, both in the composition and/or activity of the gastrointestinal microbiota that confers benefits upon host well being and health ${ }^{(6)}$. Inulin and oligofructose have been shown to inhibit growth of pathogens and to display a bifidogenic effect associated with a decrease in Bacteroides spp. and clostridia counts. These two $\beta(2-1)$ fructans differ according to their degree of polymerisation (DP). Inulin produced by extraction from chicory roots has a DP between 3 and 60, while oligofructose presenting a DP between 2 and 10 is obtained from inulin hydrolysis ${ }^{(7)}$. The bifidogenic nature of fructans is partly explained by the higher competitiveness that most bifidobacterial strains exhibit over other intestinal bacteria ${ }^{(8)}$. The DP was a determining factor in Bifidobacterium metabolism of fructans ${ }^{(9)}$. Generally, as chain length increased, the consumption of fructans by bifidobacteria decreased. Distinct preferences for chain length were observed according to bifidobacteria strains ${ }^{(10-12)}$. Specific enzymatic systems allowing hydrolysis of oligofructose only, inulin only or both could explain these differences ${ }^{(10)}$.

Prebiotics' effects have been extensively studied in animals ${ }^{(12-14)}$ and healthy adults ${ }^{(11,15,16)}$, but in vitro models may offer an interesting alternative since they are generally inexpensive to operate, easy to set up with no ethical needs ${ }^{(17)}$ and provide a dynamic overview of gut microbial activity and composition over several weeks. Effects of soluble and insoluble fibres have already been studied on the adult gut microbiota using different anaerobic fermentor systems $^{(18,19)}$, but very few studies have been focused on infant gut microbiota ${ }^{(20)}$. Indeed, there is no in vitro fermentation

\footnotetext{
Abbreviations: CFU, colony-forming units; DP, degree of polymerisation; FISH, fluorescence in situ hybridisation; HSI, high solubility inulin; P95, Orafti ${ }^{\circledR}$ P95; stab, stabilisation period; Syn, Synergy 1.

$\dagger$ Present address: Université de Brest, EA3882 Laboratoire Universitaire de Biodiversité et Ecologie Microbienne, IFR148 ScInBioS, ESMISAB, Technopôle de Brest-Iroise, 29280 Plouzané, France.

* Corresponding author: Christophe Lacroix, fax +41 4463214 03, email christophe.lacroix@ilw.agrl.ethz.ch
} 
model specifically set for formula-fed infant gut microbiota with low bifidobacteria concentrations, although such model would be advantageous for testing substrates for enhancing bifidobacteria concentration.

In the present study, a new in vitro fermentation model with immobilised infant faecal microbiota simulating the proximal colon of a formula-fed baby was established and used to investigate its application to prebiotic fructans. The present model based on recent models developed for simulating weaning infant $^{(20,21)}$ and adult ${ }^{(22)}$ was associated with a newly developed feeding medium simulating the ileal chyme reaching the proximal colon of infant below 4 month-old fed with a standard infant milk formula. Composition and activity of a formula-fed infant microbiota subjected to four prebiotic substrates were daily monitored during a 71-d continuous fermentation.

\section{Methods}

\section{Faeces collection and preparation of the bioreactor inoculum}

The faecal sample used for immobilisation was provided by a healthy young (4 month old) male infant fed from birth with a non-prebiotic-supplemented formula (Milupa Aptamil HA1, Milupa $\mathrm{GmbH}$, Friedrichsdorf, Germany) who had never received antibiotics. A fresh faecal sample $(11 \mathrm{~g})$ was scraped from the diaper, transferred to a tube containing $5 \mathrm{ml}$ of sterile, pre-reduced peptone water $(0.1 \%, \mathrm{pH} 7)$, and placed in an anaerobic jar with a $\mathrm{CO}_{2}$ generation system (Oxoid AnaeroGen TM, Oxoid AG, Basel, Switzerland) by the mother just after defecation. The faecal sample was kept in anaerobiosis, on ice and delivered to the laboratory within $1 \mathrm{~h}$. Immediately upon reception, the faecal suspension was weighed and the volume adjusted with reduced peptone water $(0.1 \%, \mathrm{pH} 7)$ under anaerobic conditions (anaerobic chamber; Coy Laboratories, Ann Arbor, MI, USA) to obtain a final faecal concentration of $20 \%(\mathrm{w} / \mathrm{v})$. This inoculum was then homogenised and centrifuged $(700 \mathrm{~g}$ for $1 \mathrm{~min}$; Biofuge Primo Heraeus, DJB Labcare Ltd, Newport Pagnell, UK) to remove large particles.

\section{Immobilisation technique}

After centrifugation, the inoculum was immobilised in 1-2 mm diameter gel beads composed of $2.5 \%$ gellan gum, $0.25 \%$ xanthan gum and $0.2 \%$ sodium citrate (v/w; SigmaAldrich Chemie GmbH, Buchs, Switzerland). This technique is based on a dispersion process in a two-phase system ${ }^{(18)}$. Briefly, the polymer solution was autoclaved, cooled to $43^{\circ} \mathrm{C}$ and inoculated aseptically with the faecal inoculum $(2 \%)$. After inoculation, the polymer solution was stirred into freshly autoclaved $\left(15 \mathrm{~min}\right.$ at $\left.121^{\circ} \mathrm{C}\right)$ commercial rapeseed oil at $43^{\circ} \mathrm{C}$ to obtain a suspension of aqueous droplets in oil. Gel beads were formed by cooling the suspension. After separation and washing, beads were hardened by soaking for $30 \mathrm{~min}$ in $\mathrm{CaCl}_{2}(0 \cdot 1 \mathrm{M})$. Beads with diameters in the $1.0-2.0 \mathrm{~mm}$ range were selected for fermentation by wet sieving. The entire process was completed under anaerobiosis in aseptic conditions within $1 \mathrm{~h}$. Gel beads $(60 \mathrm{ml})$ were then transferred to the bioreactor (Sixfors, Ismatec, Glattbrugg, Switzerland) with $140 \mathrm{ml}$ fresh nutritive medium. The entire procedure from faeces collection to the reactor inoculation was carried out under anaerobic conditions and completed within $3 \mathrm{~h}$ after defecation. A bead aliquot $(0.5 \mathrm{~g})$ was analysed just after immobilisation for viable counts.

\section{Fermentation media}

Two nutritive media were designed to simulate ileal chymes of infants fed with formula supplemented or without prebiotic substrates. Two typical infant formulas used in Europe, with a carbohydrate:N-compounds ratio of $83: 17$, SANOR 1 ORAF SYNG A (lactose $72 \mathrm{~g} / \mathrm{l}$, casein $6 \mathrm{~g} / \mathrm{l}$ and whey proteins $9 \mathrm{~g} / \mathrm{l}$ ) and SANOR 1 ORAF SYNG B (lactose $67.7 \mathrm{~g} / 1$, prebiotic $4 \cdot 3 \mathrm{~g} / 1$, casein $6 \mathrm{~g} / 1$ and whey proteins $9 \mathrm{~g} / \mathrm{l}$; Orafti, personal communication) have been used to calculate the non-prebiotic and prebiotic nutritive media compositions using a similar approach as described in Cinquin et al. ${ }^{(21)}$ with some modifications. Digestibility indices for lactose (98\%; Kien et al. ${ }^{(23)}$ ), casein $\left(98 \%\right.$; Drescher et al. $\left.{ }^{(24)}\right)$, whey proteins (80\%; Lindberg et al. ${ }^{(25)}$ ) and prebiotics (0\%) (Orafti, personal communication) were applied to each compound in order to obtain new carbohydrate:N-compounds ratios in the non-prebiotic (43:57) and prebiotic (74:26) nutritive media simulating undigested substrates present in infant ileal chymes reaching the proximal colon. The total substrate concentration (carbohydrates and $\mathrm{N}$-compounds) in the nonprebiotic medium was set at $15 \mathrm{~g} / \mathrm{l}$ leading to new concentrations of lactose $(6.4 \mathrm{~g} / \mathrm{l})$, casein $(0.5 \mathrm{~g} / \mathrm{l})$ and whey proteins $(8 \cdot 1 \mathrm{~g} / \mathrm{l})$. The concentration of $\mathrm{N}$-compounds (casein plus whey proteins) obtained after calculation for the non-prebiotic medium $(8.6 \mathrm{~g} / \mathrm{l})$ was kept constant in the prebiotic-supplemented medium. This led to an increase in the total substrate concentration of $32.6 \mathrm{~g} / 1$ (lactose $6.1 \mathrm{~g} / \mathrm{l}$, prebiotic $17.9 \mathrm{~g} / \mathrm{l}$, casein $0.5 \mathrm{~g} / \mathrm{l}$ and whey proteins $8.1 \mathrm{~g} / \mathrm{l}$ ) in the prebioticcontaining medium due to the high proportion of prebiotic theoretically available in the infant ileal chyme. Prebiotic substrates in the present study were provided by Beneo Orafti (Tienen, Belgium). Oligofructose (Orafti ${ }^{\circledR}$ P95, P95) is composed of $95 \%$ oligosaccharides with DP 2-9; oligofructoseenriched inulin (Orafti ${ }^{\circledR}$ Synergy 1, Syn) is composed of $30 \%$ oligosaccharides with DP $2-9$ and $70 \%$ inulin with DP $\geq 10$; high solubility inulin (HSI, Orafti ${ }^{\circledR} \mathrm{HSI}$ ) is composed of $60 \%$ oligosaccharides with DP $2-9$ and $28 \%$ inulin with DP $\geq 10$. Unless otherwise stated, all chemicals were obtained from Sigma-Aldrich Chemie GmbH.

The two nutritive media were supplemented with ( $\mathrm{g} / \mathrm{l}$ of distilled water): ox bile salts $(0 \cdot 05)$, porcine gastric mucin type (4.0), yeast extract (2.5), hemin (0.01), Tween 80 (1), peptone $(0.5)$, tryptone $(0.5)$, salts $\left(\mathrm{NaCl}, 4.5 ; \mathrm{KCl}, 4 \cdot 5 ; \mathrm{MgSO}_{4}\right.$ $7 \mathrm{H}_{2} \mathrm{O}, 1 \cdot 25 ; \mathrm{CaCl}_{2}, 2 \mathrm{H}_{2} \mathrm{O}, 0 \cdot 15 ; \mathrm{K}_{2} \mathrm{HPO}_{4}, 0.5 ; \mathrm{NaHCO}_{3}, 1 \cdot 5$; $\left.\mathrm{FeSO}_{4} 7 \mathrm{H}_{2} \mathrm{O}, 0 \cdot 005\right)$ and cysteine $(0 \cdot 8)$. A sterile-filtered vitamin solution was added $(0.5 \mathrm{ml} / \mathrm{l})$ separately to the autoclaved $\left(15 \mathrm{~min}, 121^{\circ} \mathrm{C}\right.$ ) medium (Michel et al. ${ }^{(26)}$ ).

Although less concentrated than in the prebiotic nutritive medium, the amount of substrates in the non-prebiotic medium $(15 \mathrm{~g} / \mathrm{l})$ was assumed to give sufficient fermentable substrates to sustain physiological bacterial growth (total bacteria in the range from 9 to $10 \log$ colony-forming units $(\mathrm{CFU}) / \mathrm{ml}$ ) and metabolic activity (at least $70 \mathrm{~mm}$ total SCFA in fermented medium) simulating an infant colonic microbial ecosystem (Mountzouris et al. ${ }^{(3)}$ ). This reduced 
level of substrates was also chosen to avoid an excess of fermentable substrates in the prebiotic medium leading to nonphysiological values. Prebiotic substrates were provided by Orafti. Unless otherwise stated, all chemicals were obtained from Sigma-Aldrich Chemie $\mathrm{GmbH}$.

\section{Fermentation procedure}

For bead colonisation, batch fermentations were first carried out in a bioreactor with a fermentation volume of $200 \mathrm{ml}$ (Sixfors, Ismatec, Glattbrugg, Switzerland) containing $30 \%$ of freshly inoculated beads (v/v). During colonisation (days 1 and 2), the fermented medium was aseptically replaced by fresh medium every $12 \mathrm{~h}$. The reactor was maintained at $37^{\circ} \mathrm{C}$, under continuous stirring (120 rpm) and anaerobic conditions by a continuous flow of pure $\mathrm{CO}_{2}$. The $\mathrm{pH}$ was adjusted to 6 by the addition of $4 \mathrm{M} \mathrm{NaOH}$. The continuous fermentation was carried out in the same reactor connected to a stirred feedstock vessel containing sterile culture medium continuously flushed with $\mathrm{CO}_{2}$ and maintained at $4^{\circ} \mathrm{C}$ and to an effluent receiving vessel. Continuous medium feeding was carried out using peristaltic pumps (Reglo analog, Ismatec, Glattbrugg, Switzerland) delivering a feed flow rate of $40 \mathrm{ml} / \mathrm{h}$ for a mean retention time in the reactor of $5 \mathrm{~h}$. This time was used to simulate the residence time in infant proximal colon, estimated for a total transit time of $23 \mathrm{~h}$ according to Sievers et al. ${ }^{(27)}$ who described a total transit time ranging from 5.4 to $36.5 \mathrm{~h}$ in formula-fed infants aged $113 \mathrm{~d}$. The fermentation was divided into eight test periods (Fig. 1). Four stabilisation periods with the non-prebiotic medium (stab 1-4: 12, 10, 8 and $8 \mathrm{~d}$, respectively) were intercalated with four prebiotic treatment periods for the three substrates to be tested, P95 (8 d), HSI (8 d) and Syn ( 8 d) including a repetition of the $\mathrm{P} 95$ treatment $(7 \mathrm{~d})$ at the end of the experiment. During the fermentation experiment, beads were collected on the last day of each treatment period and effluent samples $(13 \mathrm{ml})$ were collected daily. Plating was done immediately after collecting the samples, whereas fixed effluent samples and effluent supernatants were stored at $-80^{\circ} \mathrm{C}$ for a maximum of 5 months for fluorescence in situ hybridisation (FISH) and metabolite analyses according to Cinquin et al. ${ }^{(28)}$.

\section{Bacterial enumeration by plate counts}

Plate counts on non-selective (Wilkins Chalgren) and selective (Beeren's) media for total anaerobes and bifidobacterial populations, respectively, were performed daily on reactor effluents and on beads at the end of each test period. Major bacterial populations present in faeces and in beads (just after immobilisation and from the last day of stab 1) were enumerated on a panel of non-selective (Wilkins Chalgren and Nutrient Agar) and selective (Beeren's Agar, Lamvab, Reinforced Clostridial

\section{$\begin{array}{rlllllllll}\text { Days } & 3 & 15 & 23 & 33 & 41 & 49 & 57 & 65 & 71 \\ \text { Faeces } & 1 & 1 & 1 & 1 & 1 & 1 & 1 & 1\end{array}$ Inoculum}

Fig. 1. Schedule of the experimental colonic fermentation used to test three prebiotic substrates: P95 (oligofructose, Orafti ${ }^{\circledR}$ P95); HSI (inulin, Orafti ${ }^{\circledR}$ $\mathrm{HSI}$ ); Syn (oligofructose-enriched inulin Orafti ${ }^{\circledR}$ Synergy 1 ) in the intestinal fermentation model with immobilised infant microbiota (stab: stabilisation periods with the control fermentation medium).
Agar, Bacteroides Mineral Salt Agar, MacConkey Agar, Azide Blood Agar and Mannitol Salt Agar) media, as described by Cinquin et al. ${ }^{(21)}$. Samples were decimally diluted in reduced peptone water $(0.1 \%, \mathrm{pH} 7)$ under anaerobic conditions (anaerobic chamber; Coy Laboratories), and drops of $20 \mu \mathrm{l}$ appropriate dilutions were placed in a Petri dish. After drying of the drops, the plates were incubated aerobically or in anaerobic jars at $37^{\circ} \mathrm{C}$ for up to $5 \mathrm{~d}$. Cell counts were performed in duplicate and expressed as $\log _{10} \mathrm{CFU}$ per $\mathrm{g}$ (wet weight) of faeces, per $\mathrm{ml}$ of fermentation medium or per $\mathrm{g}$ (wet weight) gel beads.

\section{Bacterial enumeration by fluorescent in situ hybridisation}

Light microscopy was performed after completion of the continuous culture on glass slides with faecal inocula and fermentation samples from the last $4 \mathrm{~d}$ of each pseudo steady-state periods (stabilisation and treatment periods), as described by Schwiertz et al. ${ }^{(29)}$ and Cinquin et al. ${ }^{(28)}$. Different oligonucleotide Cy3-labelled probes (Microsynth $\mathrm{GmbH}$, Balgach, Switzerland), with hybridisation conditions (lysozyme treatment, buffers and hybridisation temperatures) specific for each probe as described in Cinquin et al. ${ }^{(28)}$, were used to detect the main populations: Eub338 for total bacteria ${ }^{(30)}$; Bif164 for Bifidobacterium spp. ${ }^{(31)}$; Bac303 for Bacteroides-Prevotella cluster $^{(32)}$; Lab158 for Lactobacillus and Enterococcus ${ }^{(33)}$, and Erec482 for Clostridium coccoidesEubacterium rectale group ${ }^{(34)}$. For total cell counts, $4^{\prime}, 6$-diamidino-2-phenylindole (Sigma-Aldrich Chemie $\mathrm{GmbH}$ ) was added at a final concentration of $1 \mu \mathrm{g} / \mathrm{ml}$ Citifluor ${ }^{\circledR}$ (Citifluor Ltd, London, UK), which was used as mounting medium to prevent fading of fluorescence. Cells were counted visually with an Olympus BX 60 epifluorescence microscope (Olympus Schweiz AG, Volketswil, Switzerland) on 10-well slides (Fisher Scientific SA, Wohlen, Switzerland). Bacterial concentrations were calculated from the bacterial density corresponding to fifteen annular regions to minimise the counting error due to the radial distribution of bacteria in wells as already described by Cinquin et al. ${ }^{(28)}$. Each assay was carried out in duplicate. The detection limit of the method was $\log 6 \cdot 0$ cell number per $\mathrm{ml}$ of fermentation effluents or $\mathrm{g}$ of faeces.

\section{Metabolite analyses}

HPLC (Hitachi LaChrome, Merck, Dietikon, Switzerland) analyses for SCFA (acetate, propionate, butyrate and formate), iso-acids (iso-butyrate and iso-valerate) and lactate were performed after completion of the continuous culture, with frozen supernatants of the faecal inoculum and fermentation samples $(1.5 \mathrm{ml})$ from the last $4 \mathrm{~d}$ of each pseudo steadystate treatment period, as described by Cleusix et al. ${ }^{(22)}$. Ammonia concentration was measured with an ammonia electrode (Metrohm, Herisau, Switzerland). All analyses were done in duplicate and expressed in $\mathrm{mm}$.

\section{Statistical analyses}

A one-way ANOVA was performed using SPSS 13 (SPSS Inc., Chicago, IL, USA) to test the effects of the different substrates on bacterial populations and metabolite production during the last $4 \mathrm{~d}$ of the pseudo steady-state periods. 
When significant differences were found below the probability level of 0.05 , treatment means were compared using the Tukey-Kramer's honestly significant difference test.

\section{Results}

\section{Immobilisation, bacterial diversity and stability}

Bacterial populations measured by plate counts in faeces and in beads just after immobilisation and at the end of stab 1 are presented in Table 1. Population of bifidobacteria enumerated in faeces used to inoculate the reactor was $6.2 \log \mathrm{CFU} / \mathrm{g}$ faeces, $9.9 \log \mathrm{CFU} / \mathrm{g}$ faeces for clostridia and $9.7 \log \mathrm{CFU} / \mathrm{g}$ faeces for gram-positive cocci. Immediately after immobilisation, all bacterial populations tested were detected in beads at lower concentrations than in faeces. They showed survival rates between 25 and $157 \%$, with the exception of Clostridium spp. and gram-positive cocci showing only 1 and $4 \%$ of cells surviving bead entrapment. At the end of stab 1, beads were highly colonised by bacteria. Concentrations close to the faecal ones with differences ranging from 0.2 to $0.7 \log$ units were obtained for most populations. Clostridia and bacteroides presented, however, a lower (by $1.4 \log$ units) and a much higher (by $3.2 \log$ units) concentration in beads than in the infant faeces. These results were confirmed on faecal and effluent sample populations analysed with the FISH technique (Table 2). Major bacterial groups recovered in faeces were also present in effluent samples and reached high and stable values with standard deviations equal or below $0 \cdot 3$ units for four consecutive days at the end of stab 1 .

Effect of prebiotics on bacterial populations enumerated with plate counts

Concentrations of total anaerobes and bifidobacteria were monitored in beads (at the end of each stabilisation period) and effluent samples (daily) by plate counts during the whole fermentation (Table 2 and Fig. 2). Total anaerobe concentrations were high and stable, with general means of $10 \cdot 1$ (SD 0.2) $\log$ CFU/g and 9.6 (SD 0.3) $\log$ CFU/ml in beads and effluent samples, respectively. In contrast, bifidobacterial

Table 1. Viable bacterial populations measured with plate counts in faeces (log colony-forming units (CFU)/g) and in beads (log CFU/g) just after immobilisation and at the end of the first pseudo-steady state stabilisation period of the continuous colonic fermentation

\begin{tabular}{lcccc}
\hline & & & \multicolumn{3}{c}{$\begin{array}{c}\text { Survival } \\
\text { rate (\%) }\end{array}$} & Stab 1 \\
\hline Total anaerobes & 10.7 & 7.3 & 10 & 10.2 \\
Facultative anaerobes & $8 \cdot 7$ & 6.1 & 63 & 8.9 \\
Clostridia & 9.9 & 5.6 & 1 & 8.5 \\
Bacteroides & 6.6 & 4.0 & 63 & 9.8 \\
Bifidobacteria & 6.2 & 4.0 & 158 & 5.5 \\
Coliforms & $8 \cdot 6$ & 5.6 & 25 & ND \\
Gram-positive cocci & 9.7 & 5.9 & 4 & 9.2 \\
Staphylococci & 8.2 & 5.5 & 50 & ND \\
\hline
\end{tabular}

Reported data are means of duplicate plating of single sample of faeces or beads (n 1). Immo, immobilisation; stab1, first stabilisation period.

* Calculated by the ratio of cell count in beads after Immo to theoretical cell count in beads with $100 \%$ cell survival. Theoretical cell count in beads was calculated considering a fivefold dilution from faeces to faecal cell suspension and $2 \%$ bead inoculation with faecal cell suspension.

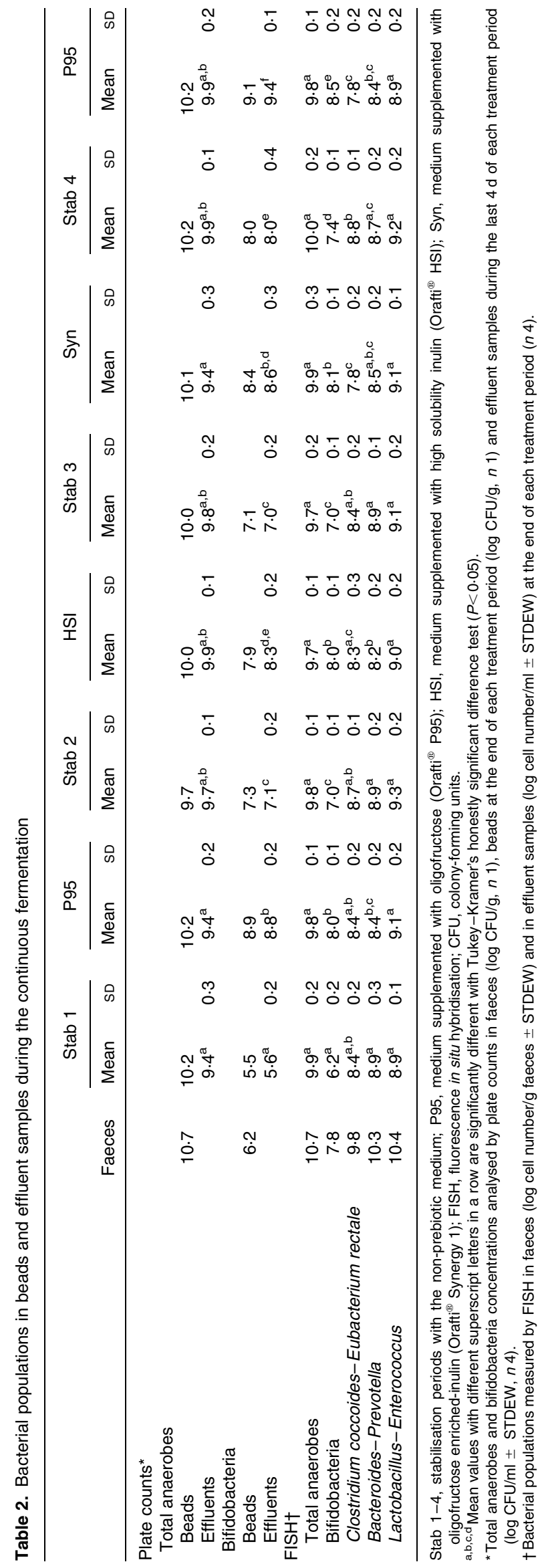




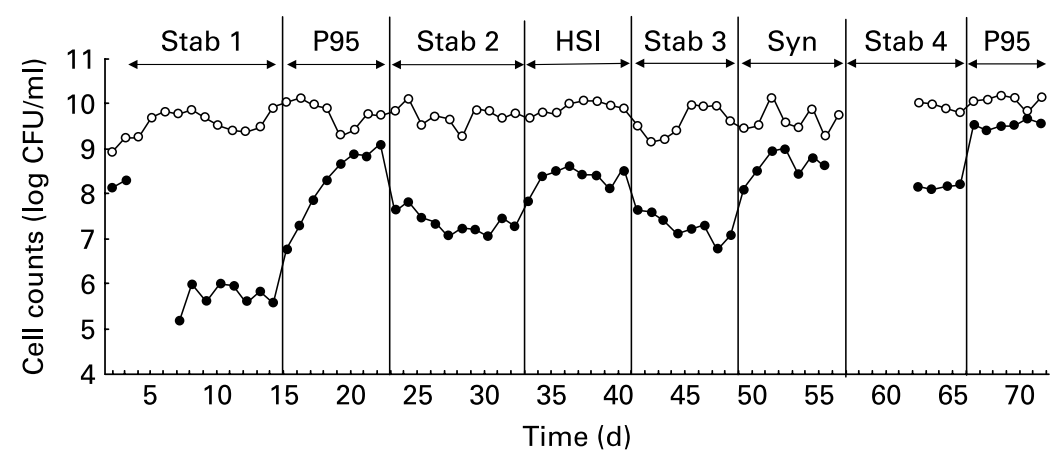

Fig. 2. Changes in total anaerobes and bifidobacteria concentrations in effluent fermentation samples during the continuous fermentation. Total anaerobes $(\mathrm{O})$, bifidobacteria (๑). Stab 1-4, stabilisation periods with the non-prebiotic medium; P95, medium supplemented with oligofructose (Orafti ${ }^{\circledR}$ P95); HSI, medium supplemented with inulin (Orafti ${ }^{\circledR} \mathrm{HSI}$ ); Syn, medium supplemented with oligofructose enriched-inulin (Orafti ${ }^{\circledR}$ Synergy 1); CFU, colony-forming units.

concentrations were strongly $(P<0 \cdot 05)$ increased during the four prebiotic treatments compared to the preceding stabilisation periods (Table 2). In effluent samples, the bifidobacterial concentrations obtained during P95 treatments (first and second) were significantly higher $(P<0.05)$ than those obtained with HSI, which were not different from Syn. These findings were confirmed in beads, which showed the same trends (Table 2). An increase in bifidobacteria counts in effluent samples over the $71 \mathrm{~d}$ of fermentation led to significantly lower and higher concentrations in stab 1 and stab 4 (5.6 and 8.0 $\log \mathrm{CFU} / \mathrm{ml}$, respectively) compared with stab 2 and stab $3(7 \cdot 1$ and $7 \cdot 0 \log \mathrm{CFU} / \mathrm{ml}$, respectively). This makes comparison of P95 with the two other prebiotics (HSI and Syn) treatments difficult.

\section{Effect of prebiotics on bacterial populations enumerated with fluorescence in situ hybridisation}

Bacterial populations were enumerated with FISH microscopy in effluent samples only (Table 2). The total bacterial population was high and stable over the whole fermentation $(9.8$ (SD 0.2) $\log$ cell number/ml). Bifidobacteria were significantly increased by $1.8 \log$ units during P95 (first treatment) and $1 \cdot 0-1 \cdot 1 \log$ units during the other three prebiotic treatments (HSI, Syn and P95 second treatment) compared to the preceding stabilisation periods. No significant differences were detected for bifidobacterial concentrations between P95 (first treatment), HSI and Syn with mean values of 8.0 (SD $0 \cdot 1$ ), 8.0 (SD 0.1) and $8 \cdot 1$ (SD 0.1) log cell number/ml, respectively. P95 (second treatment) induced a significantly higher bifidobacterial concentration (8.5 (SD 0.2) log cell number/ml) compared with the three first treatments (P95, HSI and Syn). As observed with plate counts, bifidobacterial concentrations measured with FISH gradually increased from stab 1 to stab 4, but to a lesser extent. It should be noted that bifidobacterial concentrations obtained with plate counts were systematically higher (plus $0 \cdot 3-1 \cdot 1 \log$ units) than data obtained with FISH analyses during the four prebiotic treatments.

In contrast to bifidobacteria, $C$. coccoides $-E$. rectale group and Bacteroides-Prevotella cluster slightly decreased during prebiotic treatments compared to the previous stabilisation periods. These decreases were significant $(P<0.05)$ for Bacteroides-Prevotella cluster during P95 (first treatment) and HSI, and for the $C$. coccoides $-E$. rectale group during
Syn and P95 (second treatment). No effect was observed on the lactobacilli-enterococci counts, with a mean of 9.1 (SD 0.2) $\log$ cell number/ml over the whole fermentation.

\section{Effect of prebiotic substrates on metabolic activities}

SCFA, iso-acids, lactate and ammonia concentrations were analysed in faeces and in effluent samples during the whole fermentation trial, and means were calculated for the last $4 \mathrm{~d}$ of each treatment period. The total SCFA concentration $(90.3 \mathrm{~mm})$ measured in the faecal sample was divided into $61 \%$ acetate, $34 \%$ propionate and $5 \%$ butyrate. Similar concentrations for total SCFA were tested during the four stabilisation periods (ranging from 79 to $88 \mathrm{~mm}$ ), but with different SCFA ratios (Table 3 ). The percentage of butyrate increased $(19.0(\mathrm{SD} 2.4) \%)$ and that of acetate decreased (-20.5 (SD 3.1$) \%$ ) compared to faeces whereas the percentages of propionate and formate remained constant. Iso-acids and ammonia concentrations were high in the range from 13 to $15 \mathrm{~mm}$ and from 64 to $74 \mathrm{mM}$, respectively. There were no significant differences between the four stabilisation periods for all parameter analysed, showing that the system was stable and suggesting that different treatments can be compared within the same fermentation experiment.

P95 and HSI, and Syn to a lesser extent, increased $(P<0.05)$ the fermentation capacity of the microbiota. The acetate ratio largely increased, whereas propionate and butyrate ratios decreased during prebiotic treatments (Table 3). These increases in SCFA production were concomitant with a decrease in ammonia and iso-acid concentrations and an increase in formate concentrations. The dynamic effect of prebiotics on SCFA concentrations is presented in Fig. 3 with a rapid increase in acetate concentration followed by a transient increase in lactate concentration, except for P95 (first treatment) where no increase in lactate concentration was observed.

\section{Discussion}

In the present study, we tested the effects of three different fructans (oligofructose, oligofructose-enriched inulin and inulin) during the same colonic fermentation performed with a new fermentation model simulating the proximal colon of a young formula-fed infant. 
|ㅁำ

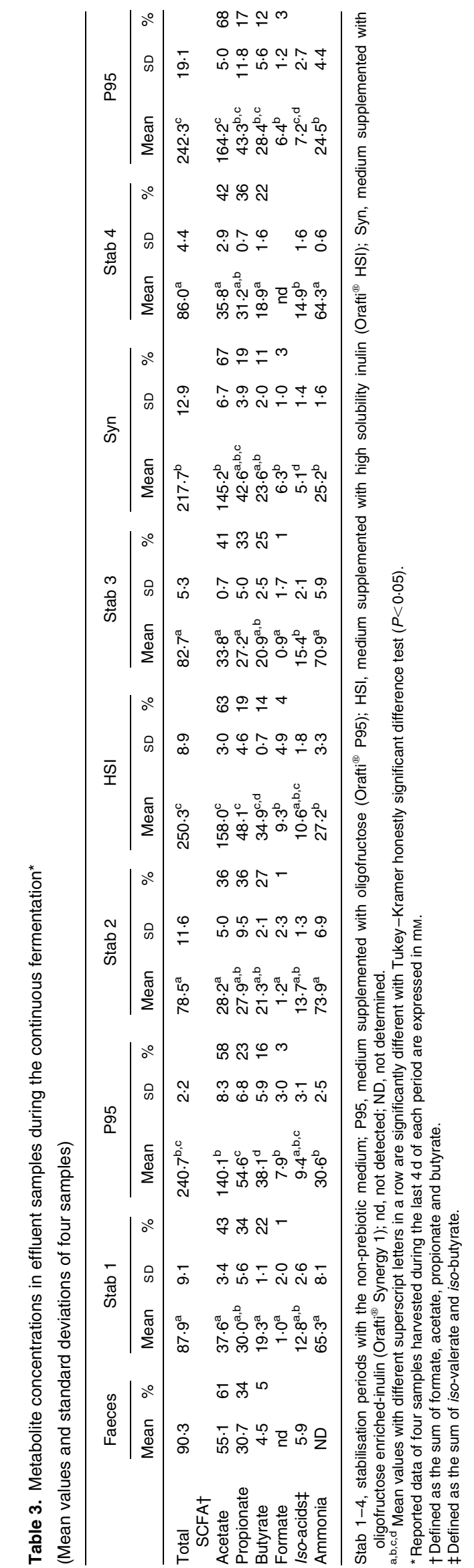

The faecal sample obtained from a 4-month-old, $100 \%$ formula-fed baby harboured high clostridia and bacteroides and low bifidobacteria concentrations with a SCFA profile dominated by acetic and propionic acids with no lactic acid and a low level of butyric acid, which is commonly met in formula-fed infants ${ }^{(3,35)}$. As already observed in a previous study (Cinquin et al. ${ }^{(21)}$ ), immobilisation allowed the recovery of the main bacterial populations. All major groups of intestinal bacteria found in faeces were present in beads and effluent samples and reached stable and similar values as in faeces at the end of the first stabilisation period.

During the four stabilisation periods, bacterial populations with highest numbers in reactor effluents were the Bacteroides-Prevotella cluster and $C$. coccoides $-E$. rectale group, which belong to the dominant populations of formula-fed infants, as described by Edwards \& Parrett ${ }^{(35)}$ and Harmsen et al. ${ }^{(36)}$. Lactobacilli and/or enterococci, detected with the oligonucleotide probe Lab158, were also present in high numbers. Contrary to infant faeces and literature data (Edwars \& Parett ${ }^{(35)}$ ), the butyrate percentages for the different stabilisation periods simulating a formula-fed baby were very high $(22-27 \%)$. This was likely a result of the high concentration of $C$. coccoides $-E$. rectale group, which harbours a high proportion of butyrate-producing bacteria ${ }^{(37)}$.

Our data for the selected medium composition and fermentation conditions ( $\mathrm{pH} 6$, mean retention time of $5 \mathrm{~h}$ ) fully met initial assumptions for total anaerobes and bifidobacteria concentrations (in the range from $10^{9}$ to $10^{10} \mathrm{CFU} / \mathrm{ml}$ and below $10^{9} \mathrm{CFU} / \mathrm{ml}$, respectively) and metabolic activity (at least $70 \mathrm{~mm}$ SCFA in the fermentation medium) in the infant model. The reduced amount of substrates $(15 \mathrm{~g} / \mathrm{l})$ in the control non-prebiotic medium provided a sufficient source of fermentable substrates to allow bacterial growth and metabolic activity comparable to that of an infant microbiota. These results on total bacterial and total metabolite concentrations measured in reactor effluents during the four stabilisation periods were in accordance with physiological values of the proximal colon of a 4-month-old formula-fed infant (total anaerobes of $8 \cdot 5-10 \cdot 5 \log \mathrm{CFU} / \mathrm{g}$ faeces $^{(29)}$ and total SCFA concentrations of approximately $70-130 \mathrm{mmol} / \mathrm{kg}$ faeces according to Mountzouris et al. ${ }^{(3)}$ ). Lactobacilli and/or enterococci, detected with oligonucleotide probe Lab158, were also present in high numbers. There were no significant differences among the four different stabilisation periods concerning bacterial and metabolite concentrations in effluent samples from the last $4 \mathrm{~d}$ of each period, except a progressive increase in bifidobacterial concentrations, which can be explained by additional colonisation of beads with bifidobacteria during prebiotic periods. To improve the accuracy of the comparison and limit this bifidobacteria counts drift in beads during successive prebiotic and stabilisation periods, it may prove useful to first increase the bifidobacterial concentration in colonised beads using a chyme-simulating medium supplemented with a prebiotic substrate, and then change the composition of the medium to produce low bifidobacteria counts in the effluent medium during stabilisation periods for comparison with prebiotic treatments. However, our present data show the good stability of the colonic model and indicate the validity of the comparison between successive treatments applied during the same fermentation experiments. 


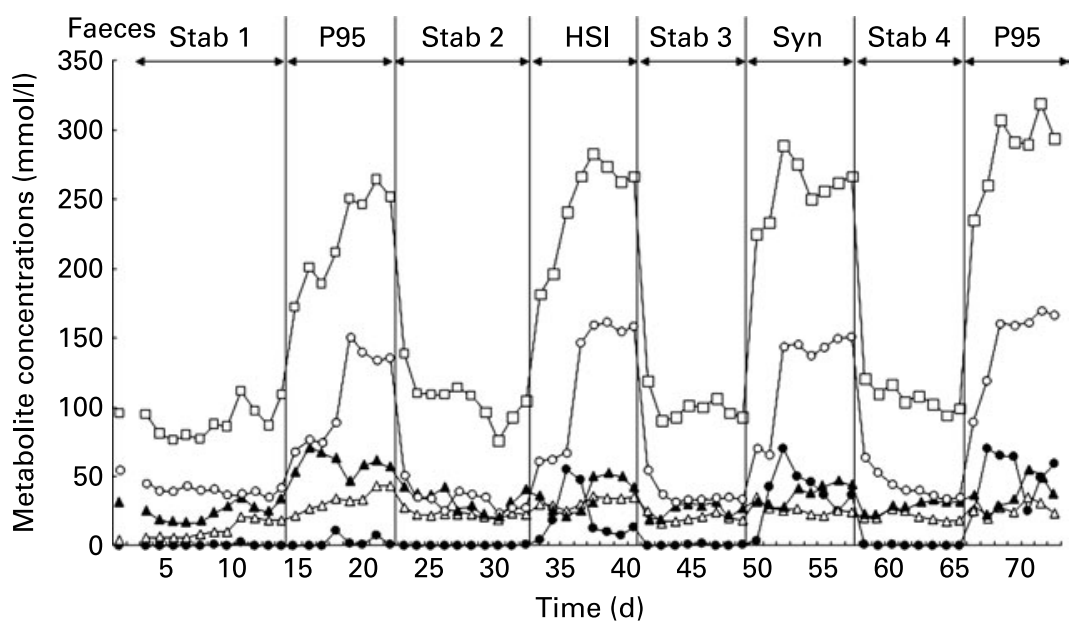

Fig. 3. Changes in metabolite concentrations ( $\mathrm{mm})$ in faeces and fermentation medium for different times and fermentation periods. Acetate $(O)$, propionate $(\mathbf{\Lambda})$, butyrate $(\Delta)$, lactate $(\bullet)$ and total SCFA $(\square)$ concentrations $(\mathrm{mm})$ in the faecal inoculum and fermentation medium. Stab 1-4, stabilisation periods with the nonprebiotic medium; P95, medium supplemented with oligofructose (Orafti ${ }^{\circledR}$ P95); HSI, medium supplemented with inulin (Orafti ${ }^{\circledR}$ HSI); Syn, medium supplemented with oligofructose enriched-inulin (Orafti ${ }^{\circledR}$ Synergy 1).

Bacteria enumeration with FISH and plate counts for total anaerobes and bifidobacteria gave similar results (Table 2). A significant increase $(P<0.05)$ in the bifidobacterial population was observed with both methods in reactor effluents for the three fructans studied to the preceding stabilisation periods, while the total anaerobes remained high and stable. Significant differences in bifidobacterial concentrations were, however, observed between the methods. The differences between FISH and plate counts may be due to different species being targeted by the two methods ${ }^{(21,31)}$. Bifidobacterium adolescentis population has been stimulated by inulin supplementation in a recent $\operatorname{study}^{(11)}$, but media for bifidobacteria such as Beeren's underestimate the number of B. adolescentis ${ }^{(38)}$. By contrast, bifidobacterial concentrations were sometimes found higher with plate counts than by FISH analysis, and selectivity of all media for bifidobacteria can be questioned ${ }^{(39)}$.

The increase in bifidobacterial counts between prebiotic treatments and preceding stabilisation periods ranged from 1.0 to $3.2 \log$ units, depending on the method of analysis and prebiotic treatment. The greatest increase was observed for the first P95 treatment, independent from the method of analysis. This is explained likely by the low counts of bifidobacteria measured during stab 1 compared with the other stabilisation periods. There is a stronger bifidogenic effect of prebiotics for low initial levels of bifidobacteria ${ }^{(40)}$. But this difference could be also explained by the different DP distribution of P95 since oligofructose could have a stronger effect ${ }^{(13)}$ or a more rapid effect on bifidobacteria increase than $\mathrm{HSI}^{(41,42)}$. Indeed, long-chain fructans with $\mathrm{DP}>10$ are fermented on average half as fast as short-chain fructans with DP $<10^{(43)}$. For all other prebiotic treatments (P95, Syn and HSI), the increase in bifidobacterial counts was similar when analysed by FISH.

Lactobacilli can also increase with prebiotics, whereas bacteroides and clostridia may decrease ${ }^{(12,44,45)}$. Data obtained for reactor effluents analysed with FISH showed a decrease in Bacteroides-Prevotella cluster and C. coccoides-E. rectale group with prebiotic treatments (although not statistically significant for all treatments). There was no significant increase in the lactobacilli-enterococci populations for any of the prebiotic substrates tested.

As expected, all four prebiotic treatments significantly increased SCFA production and decreased ammonia compared to stabilisation periods. This can be explained by the large change of carbohydrates: $\mathrm{N}$-compounds ratio between prebiotic and control treatments with about four times higher carbohydrate concentrations during prebiotic treatments in the feeding medium ${ }^{(12,28,44)}$. DP distribution did not influence the prebiotic effect of substrates in the present study contrary to Van de Wiele et al. ${ }^{(18)}$ who suggested a more pronounced in vitro prebiotic effect of fructans of longer DP. Butyrate concentrations significantly increased during prebiotic treatments, except for Syn. However, in the present study, the increase in butyrate was not specific, but due to a general increase in the fermentation activity. No increase in the butyrate ratio was observed during prebiotic treatments, probably due to an already high butyrate concentration (and ratio) present during stabilisation periods or the transformation of lactate into propionate instead of butyrate ${ }^{(12,46)}$. Lactate, as a transient metabolite, was primarily produced during the first days of the prebiotic treatments (except during the first prebiotic treatment with P95), which is typical for a fast fermentation and is probably due to a rapid increase in bifidobacteria. After a few days, the lactate-utilising bacteria colonise the infant gut and lactate decreased together with an increase in propionate. This was likely due to the fermentation of lactate to propionate and acetate at a ratio of $2: 1$, which can be done by many bacteria such as Propionibacterium spp., Veillonella spp. or Clostridium spp. ${ }^{(47)}$.

In conclusion, the present study showed that the in vitro colonic model with immobilised infant microbiota can be used to study several prebiotic substrates in the same trial with the same microbiota. All three prebiotic substrates led to significant increases in the bifidobacterial population and in metabolite production. We conclude that the established in vitro model is valid to test effects of fibre-type substrates on the infant gut flora and could be a powerful model to study diet modulation on the formula-fed infant microbiota. 


\section{Acknowledgements}

We are grateful to Beneo Orafti (Tienen, Belgium) for supplying the prebiotic substrates and financial support. G. L.-B. designed, conducted, analysed the study and drafted the paper. C. L. designed and supervised the study along with drafting the manuscript. S. B. conducted experiments and C. C. analysed data and drafted the paper.

The authors have no conflicts of interest to disclose.

\section{References}

1. Waligora-Dupriet AJ, Campeotto F, Nicolis I, et al. (2007) Effect of oligofructose supplementation on gut microflora and well-being in young children attending a day care centre. Int $J$ Food Microbiol 113, 108-113.

2. Mata LJ, Kronmal RA, Garcia B, et al. (1976) Breast-feeding, weaning and the diarrhoeal syndrome in a Guatemalan Indian village. Acute diarrhoea in childhood. Ciba Found Symp 311-338.

3. Mountzouris KC, McCartney AL \& Gibson GR (2002) Intestinal microflora of human infants and current trends for its nutritional modulation. Br J Nutr 87, 405-420.

4. Kunz C \& Rudloff S (1993) Biological functions of oligosaccharides in human milk. Acta Paediatr 83, 903-912.

5. Kunz C \& Rudloff S (2008) Potential anti-inflammatory and anti-infectious effects of human milk oligosaccharides. Adv Exp Med Biol 606, 455-465.

6. Macfarlane GT, Steed H \& Macfarlane S (2008) Bacterial metabolism and health-related effects of galacto-oligosaccharides and other prebiotics. J Appl Microbiol 104, 305-344.

7. Roberfroid MB (2007) Inulin-type fructans: functional food. J Nutr 137, 2493S-2502S.

8. Michel C (2008) Quel avenir pour le concept des prébiotiques? Bull Soc Fr Microbiol 23, 83-90.

9. Perrin S, Fougnies C, Grill JP, et al. (2002) Fermentation of chicory fructo-oligosacharides in mixture of different degrees of polymerization by three strains of bifidobacteria. Can $J$ Microbiol 48, 769-763.

10. Rossi M, Corradini C, Amaretti A, et al. (2005) Fermentation of fructooligosaccharides and inulin by bifidobacteria: a comparative study of pure and fecal cultures. Appl Environ Microbiol 71, 50-58.

11. Ramirez-Farias C, Slezak K, Fuller Z, et al. (2008) Effect of inulin on the human gut microbiota: stimulation of Bifidobacterium adolescentis and Faecalibacterium prausnitzii. Br J Nutr 101, 541-550.

12. Le Blay G, Michel C, Blottière HM, et al. (1999) Prolonged intake of fructo-oligosaccharides induces a short-term elevation of lactic acid-producing bacteria and a persistent increase in cecal butyrate in rats. $J$ Nutr 129, 2231-2235.

13. Kleessen B, Hartmann L \& Blaut M (2001) Oligofructose and long-chain inulin: influence on the gut microbial ecology of rats associated with a human faecal flora. $B r J$ Nutr 86, 291-300.

14. Demigné C, Jacobs H, Moundras C, et al. (2008) Comparison of native or reformulated chicory fructans, or non-purified chicory, on rat cecal fermentation and mineral metabolism. Eur J Nutr 47, 366-374.

15. Kolida S \& Gibson GR (2007) Prebiotic capacity of inulin-type. J Nutr 137, 2503S-2506S.

16. Kruse HP, Kleessen B \& Blaut M (1999) Effects of inulin on faecal bifidobacteria in human subjects. Br J Nutr 82, 375-382.

17. Macfarlane GT \& Macfarlane S (2007) Models for intestinal fermentation: association between food components, delivery systems, bioavailability and functional interactions in the gut. Curr Opin Biotechnol 18, 156-162.

18. Van de Wiele T, Boon N, Possemiers S, et al. (2007) Inulin-type fructans of longer degree of polymerization exert more pronounced in vitro prebiotic effects. J Appl Microbiol 102, 452-460.

19. Duncan SH, Scott KP, Ramsay AG, et al. (2003) Effects of alternative dietary substrates on competition between human colonic bacteria in an anaerobic fermentor system. Appl Environ Microbiol 69, 1136-1142.

20. Cinquin C, Le Blay G, Fliss I, et al. (2006) Comparative effects of exopolysaccharides from lactic acid bacteria and fructooligosaccharides on infant gut microbiota tested in an in vitro colonic model with immobilized cells. FEMS Microbiol Ecol 57, 324-336.

21. Cinquin C, Le Blay G, Fliss I, et al. (2004) Immobilization of infant fecal microbiota and utilization in an in vitro colonic fermentation model. Microb Ecol 48, 128-138.

22. Cleusix V, Lacroix C, Vollenweider S, et al. (2008) Glycerol induces reuterin production and decreases Escherichia coli population in an in vitro model of colonic fermentation with immobilized human feces. FEMS Microbiol Ecol 63, 56-64.

23. Kien CL, McClead RE \& Cordero L (1998) Effects of lactose intake on lactose digestion and colonic fermentation in preterm infants. J Pediatr 133, 401-405.

24. Drescher K, Roos N, Pfeuffer M, et al. (1999) Recovery of $15 \mathrm{~N}$ lactoferrin is higher than that of $15 \mathrm{~N}$-casein in the small intestine of suckling, but not adult miniature pigs. J Nutr 129, 1026-1030.

25. Lindberg T, Engberg S, Sjoberg LB, et al. (1998) In vitro digestion of proteins in human milk fortifiers and in preterm formula. J Pediatr Gastroenterol Nutr 27, 30-36.

26. Michel C, Kravtchenko TP, David A, et al. (1998) In vitro prebiotic effects of Acacia gums onto the human intestinal microbiota depends on both botanical origin and environmental $\mathrm{pH}$. Anaerobe 4, 257-266.

27. Sievers E, Oldigs HD, Schulz-Lell G, et al. (1993) Faecal excretion in infants. Eur J Pediatr 152, 452-454.

28. Cinquin C, Le Blay G, Fliss I, et al. (2006) New three-stage in vitro model for infant colonic fermentation with immobilized fecal microbiota. FEMS Microbiol Ecol 57, 340-342.

29. Schwiertz A, Le Blay G \& Blaut M (2000) Quantification of different Eubacterium spp. in human fecal samples with species-specific 16S rRNA-targeted oligonucleotide probes. Appl Environ Microbiol 66, 375-382.

30. Amann RI, Ludwig W \& Schleifer KH (1995) Phylogenetic identification and insitu detection of individual microbial cells without cultivation. Microbiol Rev 59, 143-169.

31. Langendijk PS, Schut F, Jansen GJ, et al. (1995) Quantitative fluorescence in situ hybridization of Bifidobacterium spp. with genus-specific 16S rRNA-targeted probes and its application in fecal samples. Appl Environ Microbiol 61, 3069-3075.

32. Manz W, Amann R, Ludwig W, et al. (1996) Application of a suite of $16 \mathrm{~S}$ rRNA-specific oligonucleotide probes designed to investigate bacteria of the phylum cytophaga-flavobacterbacteroides in the natural environment. Microbiology 142, 1097-1106.

33. Harmsen H, Elfferich P \& Welling GW (1999) A 16SrRNAtargeted probe for detection of Lactobacilli and Enterococci in fecal samples by fluorescent in situ hybridization. Microb Ecol Health Dis 11, 3-12.

34. Franks AH, Harmsen HJ, Raangs GC, et al. (1998) Variations of bacterial populations in human feces measured by fluorescent in situ hybridization with group-specific 16S rRNA-targeted oligonucleotide probes. Appl Environ Microbiol 64, 3336-3345.

35. Edwards CA \& Parrett AM (2002) Intestinal flora during the first months of life: new perspectives. Br J Nutr 88, 11-18. 
36. Harmsen HJM, Wildeboer-Veloo ACM, Raangs GC, et al. (2000) Analysis of intestinal flora development in breast-fed and formula-fed infants by using molecular identification and detection methods. J Pediatr Gastroenterol Nutr 30, 61-67.

37. Barcenilla A, Pryde SE, Martin JC, et al. (2000) Phylogenetic relationships of butyrate-producing bacteria from the human gut. Appl Environ Microbiol 66, 1654-1661.

38. Apajalahti JH, Kettunen A, Nurminen PH, et al. (2003) Selective plating underestimates abundance and shows differential recovery of bifidobacterial species from human feces. Appl Environ Microbiol 69, 5731-5735.

39. Hartemink R \& Rombouts FM (1999) Comparison of media for the detection of bifidobacteria, lactobacilli and total anaerobes from faecal samples. J Microbiol Methods 36, 181-192.

40. Rycroft CE, Jones MR, Gibson GR, et al. (2001) A comparative in vitro evaluation of the fermentation properties of prebiotic oligosaccharides. J Appl Microbiol 91, 878-887.

41. Stewart ML, Timm DA \& Slavin JL (2008) Fructooligosaccharides exhibit more rapid fermentation than long-chain inulin in an in vitro fermentation system. Nutr Res 28, 329-334.
42. Pompei A, Cordisco L, Raimondi S, et al. (2008) In vitro comparison of the prebiotic effects of two inulin-type fructans. Anaerobe 14, 280-286.

43. Roberfroid MB, Van Loo JA \& Gibson GR (1998) The bifidogenic nature of chicory inulin and its hydrolysis products. $J$ Nutr 128, $11-19$

44. Gibson GR \& Wang X (1994) Enrichment of bifidobacteria from human gut contents by oligofructose using continuous culture. FEMS Microbiol Lett 118, 121-127.

45. Macfarlane S, Macfarlane GT \& Cummings JH (2006) Review Article: prebiotics in the gastrointestinal tract. Aliment Pharmacol Ther 24, 701-714.

46. Duncan SH, Louis P \& Flint HJ (2004) Lactate-utilizing bacteria, isolated from human feces, that produce butyrate as a major fermentation product. Appl Environ Microbiol 70, 5810-5817.

47. Seeliger S, Janssen PH \& Schink B (2002) Energetics and kinetics of lactate fermentation to acetate and propionate via methylmalonyl-CoA or acrylyl-CoA. FEMS Microbiol Lett 211, 65-70. 